\title{
Enterprise product sales market selection model
}

\author{
Vladimir Anisimov ${ }^{1, *}$, Evgeniy Anisimov ${ }^{2}$, and Tatyana Saurenko ${ }^{2}$ \\ ${ }^{1}$ Peter the Great St. Petersburg Polytechnic University (SPbPU), Graduate School of Business and \\ Management, Polytechnicheskaya, 29, St. Petersburg, 195251, Russian Federation \\ ${ }^{2}$ Peoples Friendship University of Russia (RUDN University), 6 Miklukho-Maklaya St, Moscow, \\ 117198, Russian Federation
}

\begin{abstract}
Efficient sales logistics is the foundation of sustainability of enterprises in the real economy. This is due to the fact that it is the closing link in the logistics system, production of goods and provides a choice of distribution channels and their participants. The choice of distribution channels, first of all, involves the determination of markets for the products manufactured by the enterprise. Due to the need to ensure the effectiveness of this choice of taking into account a large number of various factors, the solution to this problem involves the use of formalized models and techniques. The article proposes a model and methodology that provides a reasonable choice of markets for the products of an enterprise in the real sector of the economy at the initial stage of forming its marketing logistics strategy. The model is based on a formal apparatus of statistical conclusions based on nonparametric statistics. This made it possible to take into account the inherent uncertainty of the source information inherent in the considered selection problem. The proposed model may be useful for digitalization in the field of logistics management of enterprises in the real sector of the economy.
\end{abstract}

\section{Introduction}

The most important element of enterprise management in the real sector of the economy is the formation of a sales logistics strategy [1 - 5]. Its formation, first of all, involves the choice of markets for manufactured products. The solution to this problem often has to be carried out in conditions of significant uncertainty. The level of uncertainty is characterized by the information situation in which the choice is made [6 - 8]. A typical information situation is the lack of reliable quantitative assessments of possible markets for enterprises. In such a situation, one has to rely on many of their qualitative characteristics, often exhausted by the ability to rank markets according to some preference criteria. The components of the estimation vectors are the serial numbers of the markets in a series of preferences for the established indicators. The formation of a model for substantiating the choice of a market for manufactured products in this information situation is the purpose of this article.

\footnotetext{
* Corresponding author: an-33@yandex.ru
} 


\section{Formalized representation of the model}

In the basis of the formalization of the problem of choosing the sales market for the products manufactured by the enterprise in the situation under consideration, the mathematical apparatus of statistical conclusions can be used. It is based on nonparametric statistics. The considered problem of choice in a formalized representation is as follows.

Known:

1) Possible markets for the company's products

$$
J=\{1,2, \ldots, J\} .
$$

2) Indicators by which markets are compared

$$
I=\{1,2, \ldots, I\} .
$$

3) It is believed that the information available for a comparative assessment of markets is limited to the possibility of ranking them according to established indicators.

It is required from the set (1) based on indicators (2) to select the most rational market for the sale of enterprise products.

In a formalized view, the ranking of possible markets for the company's products consists in building a matrix

$$
A=\left\|r_{i j}\right\|, \quad i=1,2, \ldots, I, \quad j=1,2, \ldots, J
$$

where ${ }^{r_{i j}}$ serial number (rank) of the j-th market, compared by the i-th indicator;

$\mathrm{J}$ - is the number of compared markets for products;

I - is the number of established (or available) quality indicators of each market.

In the interests of an objective comparison of markets from the set (1) in the information situation under consideration, it is advisable to use methods of the theory of statistical conclusions based on nonparametric statistics [ ].

As a generalized characteristic of each market in the considered information situation, the rank correlation coefficients [9 - 11] can be used. They are determined by the relations

$$
\rho_{j}=1-\frac{\sum_{i=1}^{I}\left(r_{i j}-1\right)}{I(J-1)}, \quad j=1,2, \ldots, J .
$$

Moreover, $\rho_{j}=1$ corresponds to the best, and $\rho_{j}=0$ - to the worst of the compared markets for the company's products.

Since the ranks of the markets from the set (1) are established on the basis of the experience of the persons performing the ranking, the correlation coefficients can be considered as random variables. Therefore, when conducting a comparative analysis of product sales markets, it is advisable to evaluate possible errors of the first kind (reject the hypothesis of the best option, if it is true).

Based on the nature of the problem being solved, the domain $[0,1]$ is the domain of random variables $\rho_{j}(\mathrm{j}=1,2, \ldots, \mathrm{J})$. A good statistical model for random variables whose values are limited by the interval $[0,1]$ is the $\beta$-distribution. Therefore, in the current information situation, it can be assumed that the coefficients $\rho_{j}(\mathrm{j}=1,2, \ldots, \mathrm{J})$ of the rank correlation of the compared sales markets of the enterprise's products are random variables distributed in accordance with the $\beta$ - distribution. Their distribution functions are determined by the relations 


$$
P\left(\rho_{j}<x\right)=\frac{1}{B\left(a_{j}, b_{j}\right)} \int_{0}^{x} t^{a_{j}-1}(1-t)^{b_{j}-1} d t, \quad j=1,2, \ldots, J
$$

where $a_{j}, b_{j}-\beta$ distribution parameters;

$B\left(a_{j}, b_{j}\right)$ - Euler B-function.

The parameters of the $\beta$-distribution form for the coefficients $\rho_{j}(\mathrm{j}=1,2, \ldots, \mathrm{J})$ of the rank correlation of the compared sales markets of the enterprise's products are related to the mathematical expectation and dispersion of a random variable by the dependencies:

$$
\begin{gathered}
M_{j}=\frac{a_{j}}{a_{j}+b_{j}}, \quad j=1,2, \ldots, J, \\
D_{j}=\frac{a_{j} b_{j}}{\left(a_{j}+b_{j}\right)^{2}\left(a_{j}+b_{j}+1\right)}, \quad j=1,2, \ldots, J .
\end{gathered}
$$

Solving (6), (7) relatively $a_{j}, b_{j}$, we obtain

$$
\begin{aligned}
& a_{j}=\frac{M_{j}^{2}\left(1-M_{j}\right)}{D_{j}}-M_{j}, \quad j=1,2, \ldots, J \\
& b_{j}=\frac{M_{j}}{D_{j}}\left(1-M_{j}\right)^{2}-\left(1-M_{j}\right), \quad j=1,2, \ldots, J .
\end{aligned}
$$

Substituting the values $S_{j}$ from (10) into these relations, taking into account the fact that the ranks $r_{i j}(\mathrm{j}=1,2, \ldots, \mathrm{J})$ are sequences of numbers from unity to $\mathrm{J}$, we obtain

$$
M_{j}\left(s_{j}\right)=\frac{1}{J} \sum_{j=1}^{J} \sum_{i=1}^{I}\left(r_{i j}-1\right)=\frac{1}{J} \sum_{i=1}^{I} \sum_{j=1}^{J}\left(r_{i j}-1\right)=\frac{I}{J} \sum_{j=1}^{n}\left(r_{i j}-1\right)=\frac{I(J-1)}{2}
$$

For the variance of a quantity $S_{j}$ by similar transformations, we obtain

$$
D_{j}\left(s_{j}\right)=\frac{\sum_{j=1}^{J} S_{j}^{2}}{J}=\frac{I^{2}(J-1)^{2}}{4} .
$$

Given the obtained relations, the mathematical expectation and variance of the coefficients $\rho_{j}(\mathrm{j}=1,2, \ldots, \mathrm{J})$ of the rank correlation of the considered sales markets are respectively equal

$$
\begin{gathered}
M_{j}=1-\frac{M_{j}\left(s_{j}\right)}{I(J-1)}=\frac{1}{2}, \\
D_{j}=\frac{\sum_{j=1}^{n} S_{j}^{2}}{I^{2} J(J-1)^{2}}-\frac{1}{4}
\end{gathered}
$$

Substituting (12), (13) in (8), (9) we find the values of the $\beta$-distribution form parameters for the rank correlation coefficients of the considered sales markets of the enterprise's products: 


$$
a_{j}=b_{j}=\frac{1}{2}\left\{\frac{\left[I^{2} J(J-1)^{2}\right]}{4 \sum_{j=1}^{n} S_{j}^{2}-I^{2} J(J-1)^{2}}-1\right\}, \quad j=1,2, \ldots, J
$$

To determine the critical value of the coefficient ${ }^{x_{j}}$ of rank correlation by a given level of significance (probability of an error of the first kind) $\gamma=1-P$, we use the relation

$$
x_{j}=M_{j}+\psi(P)\left[M_{j}\left(1-M_{j}\right)\right]^{\frac{1}{2}}\left(a_{j}+b_{j}\right)^{-\frac{1}{2}}+\left[\psi^{2}(P)-1\right] \frac{1-2 M_{j}}{3} \frac{1}{a_{j}+b_{j}},
$$

where $\psi(P)$ - $\mathrm{P}$ - quantile of the normal distribution.

The physical meaning of $\mathrm{P}$ - quantile is determined by the relation $F(\psi<\psi(P))=P$. It means that if the P-quantile of a random variable $\psi$, with the distribution law $\mathrm{F}$, then the probability that $\psi$ does not exceed the value is equal to $P$.

Substituting (12) and (14) into (15) and considering that $P=1-\gamma$, we obtain

$$
x_{j}=\frac{1}{2}\left[1+\psi(1-\gamma)\left(\frac{4 R-I^{2} J(J-1)^{2}}{2 I^{2} J(J-1)^{2}-4 R}\right)^{\frac{1}{2}}\right],
$$

where $R=\sum_{j=1}^{J} S_{j}^{2}$.

The application of the proposed model is illustrated by an example. Suppose there are four possible markets for the company's products. Each project is characterized by eight quality indicators $\mathrm{Qi}, \mathrm{i}=1,2, \ldots, 8$. The ranked series of preferences of these indicators for various projects are:

$$
\begin{aligned}
& Q_{1}: q_{1}>q_{2}>q_{3}>q_{4} ; \\
& Q_{2}: q_{2}>q_{4}>q_{1}>q_{3} ; \\
& Q_{3}: q_{3}>q_{2}>q_{1}>q_{4} ; \\
& Q_{4}: q_{4}>q_{1}>q_{2}>q_{3} ; \\
& Q_{5}: q_{1}>q_{3}>q_{2}>q_{4} ; \\
& Q_{6}: q_{2}>q_{1}>q_{4}>q_{3} ; \\
& Q_{7}: q_{4}>q_{1}>q_{3}>q_{2} ; \\
& Q_{8}: q_{2}>q_{4}>q_{3}>q_{1},
\end{aligned}
$$

where qj is the identifier of the $\mathrm{j}$-th market.

Based on the ranking results, we construct a ranking matrix:

$$
A=\left\|r_{i j}\right\|=\left\|\begin{array}{llll}
1 & 2 & 3 & 4 \\
3 & 1 & 4 & 2 \\
3 & 2 & 1 & 4 \\
2 & 3 & 4 & 1 \\
1 & 3 & 2 & 4 \\
2 & 1 & 4 & 3 \\
2 & 4 & 3 & 1 \\
4 & 1 & 3 & 2
\end{array}\right\|
$$

Using formula (4) for the compared markets, we determine the rank correlation coefficients. Their values are given in table 1 . 
Table 1. Correlation coefficients of sales markets of enterprise products.

\begin{tabular}{|c|c|c|c|c|}
\hline $\begin{array}{c}\text { Product } \\
\text { Sales Mar- } \\
\text { kets }\end{array}$ & $q_{1}$ & $q_{2}$ & $q_{3}$ & $q_{4}$ \\
\hline $\begin{array}{c}\text { Correla- } \\
\text { tion coeffi- } \\
\text { cients } \rho_{j}\end{array}$ & 0.5833 & 0.7251 & 0.3333 & 0.5417 \\
\hline
\end{tabular}

From the table. 1 it follows that the largest value of the correlation coefficient $\rho 2=0.7251$ corresponds to the sales market with identifier q2.

To estimate the probability of error of the first kind $\gamma$, we use the data obtained as a result of calculations according to formula (16) under the condition that $\mathrm{J}=4, \mathrm{I}=8$, $\mathrm{R}=102+92+162+132=606$.

The calculation results are given in table. 2 .

Table 2. Calculation results.

\begin{tabular}{|c|c|c|c|c|c|}
\hline$\gamma$ & 0.14 & 0.12 & 0.10 & 0.08 & 0.06 \\
\hline$x_{j}$ & 0.723 & 0.745 & 0.767 & 0.793 & 0.823 \\
\hline
\end{tabular}

An analysis of the critical values of the probability of an error of the first kind allows us to conclude that for the best q2 coefficient of correlation in the sales market of an enterprise's products q2, the probability of an error of the first kind $\gamma$ does not exceed 0.12 . Therefore, within the framework of the considered example, this market can be considered preferable for the sale of enterprise products.

\section{Conclusions}

In general, the proposed model provides a comparative assessment of the markets for the products of an enterprise in the real sector of the economy. It allows you to take into account the information situation that is characteristic of the initial stages of the formation of the strategy of its sales logistics. The proposed model is quite simple and has significant commonality. This makes it useful for digitalizing the process of forming the company's sales logistics.

\section{References}

1. D. Waters, Logistics, An Introduction to Supply Chain Management (USA: JohnWileyandSons, 2015)

2. E.G. Anisimov, T.N. Saurenko, M.R. Gapov, E.S. Rodionova, The model for determining rational inventory in occasional demand supply chains, International Journal of Supply Chain Management 8, 1, 86-89 (2019)

3. P. Du, L. Xu, Q. Chen, S. Tsai, Pricing competition on innovative product between innovator and entrant imitator facing strategic customers, International J. of Production Research 1-19 (2016)

4. J. Du, Developing a Conceptual Framework of International Logistics Centres, 12th World Conference on Transport Research, Lisbon (2010)

5. V.S. Lukinskiy, V.V. Lukinskiy, N.G. Pletnev, Logistics and supply chain management (2016) 
6. V.G. Anisimov, E.G. Anisimov, T.N. Saurenko, M.A. Sonkin., The model and the planning method of volume and variety assessment of innovative products in an industrial enterprise, J. Phys.: Conf. Ser. 803(1), 012006 (2017) DOI: 10.1088/17426596/803/1/012006

7. T. Saurenko, E. Anisimov, V. Anisimov, A. Levina, Comparing investment projects of innovative developing strategies of municipalities, based on a set of indicators, MATEC Web of Conferences C. 01038 (2018)

8. E.G. Anisimov, V.G. Anisimov, M.A. Sonkin, Mathematical simulation of adaptive allocation of discrete resources, Proceedings of the 2016 Conference on Information Technologies in Science, Management, Social Sphere and Medicine, ITSMSSM 2016. ACSR: Advances in Computer Science Research 282-285 (2016)

9. M. Hollander, D.A. Wolfe, E. Chicken, Nonparametric Statistical Methods, John Wiley \& Sons (2014)

10. E.L. Lehmann, Nonparametrics Statistical Methods Based on Ranks (Springer-Verlag New York, 2006)

11. J.H. Albert, Bayesian estimate of the coefficient of polychoric correlation, Journal Statistical Computing and Modeling 44: 47-61 